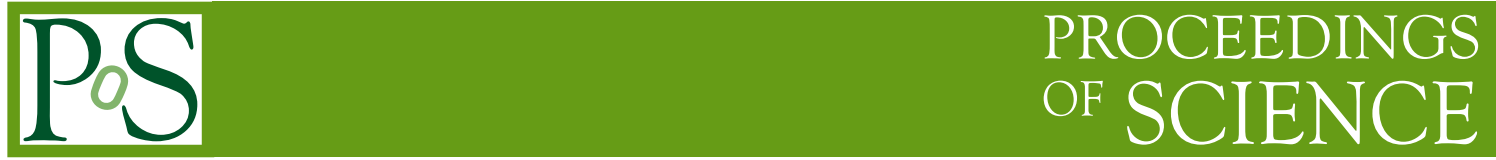

\title{
Experimental prospects for muon physics at a neutrio factory
}

\author{
B. Lee Roberts*广 \\ Department of Physics \\ Boston University \\ Boston, MA 02216 USA E-mail: roberts@bu . edu
}

Selected experiments in muon physics would add a strong component to the scientific program at a neutrio factory. The muon fluxes that could be available at such a facility would permit a search for a permanent electric dipole moment of the muon at the $\leq 10^{-25} e$-cm level, thus presenting the unique opportunity to search for an EDM in a second-generation particle. The muon to electron coherent conversion in the field of a nucleus could be searched for, and perhaps studied, down to a branching fraction of $\sim 10^{-20}$.

10th International Workshop on Neutrino Factories, Super beams and Beta beams

June 30 - July 52008

Valencia, Spain

\footnotetext{
${ }^{*}$ Speaker.

${ }^{\dagger}$ This work supported in part by the US National Science Foudation.
} 


\section{Introduction}

The muon was first observed in a Wilson cloud chamber by Kunze[1] in 1933, and identified in 1936 by Anderson and Neddermeyer[2]. The Yukawa theory of the nuclear force had predicted such a particle, but this "mesotron" as it was called, interacted too weakly with matter to be the carrier of the strong force. Today we understand that the muon was the first second-generation particle to be discovered, which with its neutrino forms the second generation in the lepton sector. Its mass, $\sim 207$ times the electron's, makes the muon magnetic interaction, along with its decays, ideal places to search for physics beyond the standard model by observing quantities which are sensitive to heavier mass scales through virtual loops. Its long lifetime, $\sim 2.2 \mu \mathrm{s}$, permits precision measurements of its properties.

In the 72 years since the muon's discovery, it has helped us learn many of nature's subatomic secrets: the parity violating $V-A$ nature of the weak interaction; the strength of the weak interaction $G_{F}$; the VEV of the Higgs field which is related to $G_{F}$; information on the modification of the weak force in the atomic nucleus that is complementary to that obtained from nuclear $\beta$ decay; and constraints on physics beyond the standard model provided by the measurement of the muon's anomalous magnetic moment.

The muon is of interest to this conference as the companion to the muon neutrino in pion decay, or as the source of a neutrino beam in the muon storage ring at a neutrino factory. The purpose of this talk is to make the case that a vigorous program of selected muon experiments must be a part of the physics program at such a facility. The neutrino factory will produce an unprecedented number of muons, and with proper planning, an intense beam could also be developed for fundamental experiments that cannot be done elsewhere. Significant additional detail is contained in a CERN working group report[15], and in the "International Scoping Study" report[16].

Three experiments, dubbed the "muon trio" by Yoshitaka Kuno, come to mind: The electric and magnetic dipole moments of the muon, and the search for neutrinoless conversion of a muon to an electron. The latter two experiments require the exceptional muon flux that could be possible at a neutrino factory.

\section{Dipole Moments}

The electric and magnetic dipole moments have been an integral part of relativistic electron (lepton) theory since Dirac's famous 1928 paper, where he pointed out that an electron in external electric and magnetic fields has "the two extra terms

$$
\frac{e \hbar}{c}(\sigma, \mathbf{H})+i \frac{e \hbar}{c} \rho_{1}(\sigma, \mathbf{E}),
$$

... when divided by the factor $2 m$ can be regarded as the additional potential energy of the electron due to its new degree of freedom[4]." These terms represent the magnetic dipole (Dirac) moment and electric dipole moment interactions with the external magnetic and electric fields.

In modern notation, the magnetic dipole moment (MDM) interaction becomes

$$
\bar{u}_{\mu}\left[e F_{1}\left(q^{2}\right) \gamma_{\beta}+\frac{i e}{2 m_{\mu}} F_{2}\left(q^{2}\right) \sigma_{\beta \delta} q^{\delta}\right] u_{\mu}
$$


where $F_{1}(0)=1$, and $F_{2}(0)=a_{\mu}$. The electric dipole moment (EDM) interaction is

$$
\bar{u}_{\mu}\left[\frac{i e}{2 m_{\mu}} F_{2}\left(q^{2}\right)-F_{3}\left(q^{2}\right) \gamma_{5}\right] \sigma_{\beta \delta} q^{\delta} u_{\mu}
$$

where $F_{2}(0)=a_{\mu}, F_{3}(0)=d_{\mu}$, with

$$
d_{\mu}=\left(\frac{\eta}{2}\right)\left(\frac{e \hbar}{2 m c}\right) \simeq \eta \times 4.7 \times 10^{-14} e \mathrm{~cm} .
$$

(This $\eta$, which is the EDM analogy to $g$ for the MDM, should not be confused with the Michel parameter $\eta$.)

The existence of an EDM implies that both $P$ and $T$ are violated[5, 6]. This can be seen by considering the non-relativistic Hamiltonian for a spin one-half particle in the presence of both an electric and magnetic field: $\mathscr{H}=-\vec{\mu} \cdot \vec{B}-\vec{d} \cdot \vec{E}$. The transformation properties of $\vec{E}, \vec{B}, \vec{\mu}$ and $\vec{d}$ are given in the Table 1 (a), and we see that while $\vec{\mu} \cdot \vec{B}$ is even under all three, $\vec{d} \cdot \vec{E}$ is odd under both $P$ and $T$. While parity violation has been observed in many weak processes, direct $T$ violation has only been observed in the neutral kaon system[7]. In the context of CPT symmetry, an EDM implies $C P$ violation, which is allowed by the standard model for decays in the neutral kaon and $B$-meson sectors.

Table 1: (a) Transformation properties of the electric and magnetic fields, and the dipole moments.

(b) Present limits on the electric dipole moments

(b)

(a)

\begin{tabular}{cccc}
\hline & $\vec{E}$ & $\vec{B}$ & $\vec{\mu}$ or $\vec{d}$ \\
\hline$P$ & - & + & + \\
$C$ & - & - & - \\
$T$ & + & - & - \\
\hline
\end{tabular}

\begin{tabular}{ccc}
\hline Particle & $\begin{array}{c}\text { Present EDM } \\
\text { Limit }(e \mathrm{~cm})\end{array}$ & $\begin{array}{c}\text { Standard Model } \\
\text { Value }(e \mathrm{~cm})\end{array}$ \\
\hline$n$ & $2.9 \times 10^{-26}(90 \% \mathrm{CL})[9]$ & $10^{-31}$ \\
\hline$e^{-}$ & $\sim 1.6 \times 10^{-27}(90 \% \mathrm{CL})[10]$ & $10^{-38}$ \\
\hline$\mu$ & $1.8 \times 10^{-19 \dagger}(\mathrm{E} 821)$ & \\
\hline${ }^{199} \mathrm{Hg}$ & $2.1 \times 10^{-28}(95 \% \mathrm{CL})[12]$ & \\
\hline
\end{tabular}

Observation of a non-zero electron or muon EDM would be a clear signal for new physics. To date no permanent EDM has been observed for the electron, the neutron, or an atomic nucleus, with the experimental limits given in Table 1(b). It is interesting to note that in his original paper[4] Dirac stated "The electric moment, being a pure imaginary, we should not expect to appear in the model. It is doubtful whether the electric moment has any physical meaning, since the Hamiltonian ...that we started from is real, and the imaginary part only appeared when we multiplied it up in an artificial way in order to make it resemble the Hamiltonian of previous theories."

While $C P$ violation is widely invoked to explain the baryon-antibaryon asymmetry of the universe, the $C P$ violation observed to date in the neutral kaon, and in the $B$ meson sectors is too small to explain it. This $C P$ deficit has motived a broad program of searches for EDMs in a range of systems. Many extensions to the standard model, such as supersymmetry, do not forbid new sources of $C P$-violation, and the failure to observe it has placed severe restrictions on many models. 


\subsection{Measurement of Dipole Moments}

Measurement of the magnetic anomaly, and a search for an EDM uses the spin motion in a magnetic field. For a muon moving in a magnetic field, the spin rotates relative to the momentum with the frequency

$$
\vec{\omega}_{a}=-\frac{q}{m}\left[a_{\mu} \vec{B}-\left(a_{\mu}-\frac{1}{\gamma^{2}-1}\right) \frac{\vec{\beta} \times \vec{E}}{c}\right] .
$$

The magnetic field in Eq. 2.5 is the average field seen by the ensemble of muons. The experiment is operated at the "magic $\gamma=29.3$ " where the second term vanishes and permits an electric quadrupole field to be used for vertical focusing. The present precision of the $a_{\mu}$ is $\pm 0.54 \mathrm{ppm}$ [3], which is discussed in a parallel paper in these proceedings[14].

The muon spin motion is modified by the presence of an electric dipole moment. The total frequency becomes $\vec{\omega}=\vec{\omega}_{a}+\vec{\omega}_{\eta}$, where

$$
\vec{\omega}_{\eta}=-\frac{q}{m}\left[\frac{\eta}{2}\left(\frac{\vec{E}}{c}+\vec{\beta} \times \vec{B}\right)\right],
$$

with $\eta$ defined by Eq. 2.4, and $\omega_{a}$ by Eq. 2.5. The spin motion resulting from the motional electric field, $\vec{\beta} \times \vec{B}$ is the dominant effect, so $\omega_{\eta}$ is transverse to $\vec{B}$. An EDM would have two effects on the precession, there would be a slight tipping of the precession plane, which would cause a vertical oscillation of the centroid of the decay electrons that out of phase with the $\omega_{a}$ precession; and the observed frequency $\omega$ would be larger,

$$
\omega=\sqrt{\omega_{a}^{2}+\left(\frac{q \eta \beta B}{2 m}\right)^{2}} .
$$

To reduce systematic errors in the muon EDM measurement, a "frozen spin" technique has been proposed[21] which uses a radial electric field in a muon storage ring, operating at $\gamma<<\gamma_{\text {magic }}$ to cancel the $(g-2)$ precession. The EDM term, Eq. 2.6, would then cause the spin to steadily move out of the plane of the storage ring. Electron detectors above and below the storage region would detect a time-dependent up-down asymmetry that increased with time. As in the $(g-2)$ experiments, detectors placed in the plane of the beam would be used, in this case to make sure that the radial- $E$-field cancels the normal spin precession exactly. Adelmann and Kirsh[22] have proposed that one could reach a sensitivity of $5 \times 10^{-23} e-\mathrm{cm}$ with a small storage ring at PSI. A letter of intent at J-PARC[23] suggested that one could reach $<10^{-24} e-\mathrm{cm}$ there. The ultimate sensitivity would need a muon intensity only available at a neutrino factory.

\section{Muon Flavor Violating Experiments}

The standard-model gauge bosons do not permit leptons to mix with each other, unlike the quark sector where mixing has been known for many years. Quark mixing was first proposed by Cabibbo[24], and extended to three generations by Kobayashi and Maskawa[25], which is described by a $3 \times 3$ mixing matrix now universally called the CKM matrix. With the discovery of neutrino mass, we know that lepton flavor violation (LFV) certainly exists in the neutral lepton 
sector, with the determination of the mixing matrix for the three neutrino flavors having become a world-wide effort.

While the mixing observed in neutrinos does predict some level of charged lepton mixing, it is many orders of magnitude below present experimental limits[15]. New dynamics[27, 28, 29, $30,31,32,33,34,36,35]$, e.g. supersymmetry, do permit leptons to mix, and the observation of standard-model forbidden processes such as

$$
\mu^{+} \rightarrow e^{+} \gamma ; \mu^{+} \rightarrow e^{+} e^{+} e^{-} ; \mu^{-} N \rightarrow e^{-} N ; \mu^{-}+\mathscr{N} \rightarrow e^{+}+\mathscr{N}^{\prime} ; \mu^{+} e^{-} \rightarrow \mu^{-} e^{+}
$$

would clearly signify the presence of new physics. The present limits on lepton flavor violation are shown in Fig. 1(a).

If lepton mixing occurs via supersymmetry, there will be a mixing between the supersymmetric leptons (sleptons) which would also be described by a $3 \times 3$ mixing matrix. The schematic connection between lepton flavor violations and the dipole moments is shown in Fig. 1(b), and there are models that try to connect these processes[37].

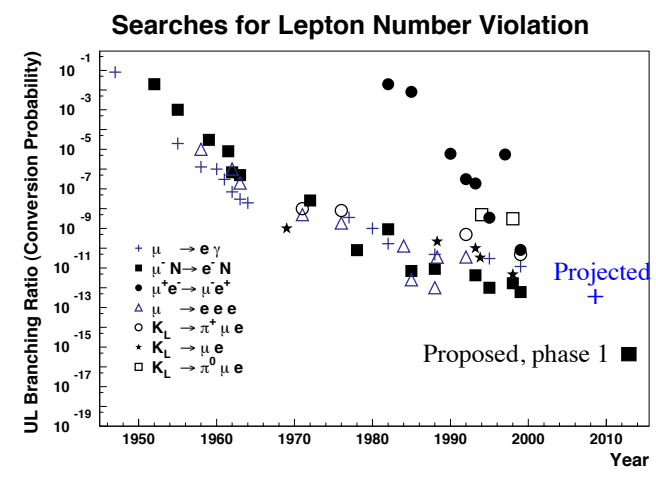

(a)

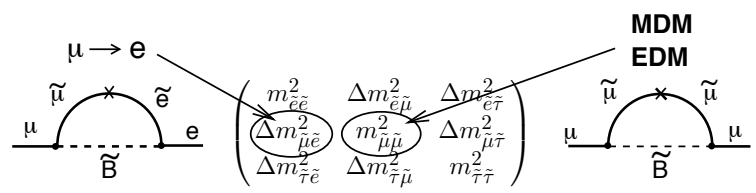

(b)

Figure 1: (a) Historical development of the 90\% C.L. upper limits on branching ratios respectively conversion probabilities of muon-number violating processes which involve muons and kaons. Also shown is the projected goal of the MEG $\left(\mu^{+} \rightarrow e^{+} \gamma\right)$ experiment which is underway at PSI, and the projected sensitivity of the proposed Mu2e experiment at Fermilab and the COMET experiment at J-PARC. (b) The supersymmetric contributions to the anomaly, and to $\mu \rightarrow e$ conversion, showing the relevant slepton mixing matrix elements. The MDM and EDM give the real and imaginary parts of the matrix element respectively.

In a large class of models, if the $\Delta \ell=1 L F V$ decay goes through the transition magnetic moment, one finds[15]

$$
\frac{B(\mu N \rightarrow e N)}{B(\mu \rightarrow e \gamma)}=2 \times 10^{-3} B(A, Z)
$$

where $B(A, Z)$ is a coefficient of order 1 for nuclei heavier than aluminum[38]. For other models, these two rates can be the same[15], so in the design of new experiments, the reach in single event sensitivity for the coherent muon conversion experiments needs to be several orders of magnitude smaller than for $\mu \rightarrow e \gamma$ to probe the former class of models with equal sensitivity. Detailed calculations of $\mu-e$ conversion rates as a function of atomic number have also been carried out[39], and if observed, measurements should be carried out in several nuclei. 
From the experimental side, the next generation $\mu \rightarrow e \gamma$ experiment, MEG, is now under way at PSI[40], with a sensitivity goal of $10^{-13}-10^{-14}$. Since the decay occurs at rest, the photon and positron are back-to-back, and share equally the energy $m_{\mu} c^{2}$. This experiment makes use of a unique "COBRA" magnet which produces a constant bending radius for the mono-energetic $e^{+}$, independent of its angle. The photon is detected by a large liquid Xe scintillation detector.

Of the various lepton-flavor violating reactions, only coherent muon conversion does not require coincidence measurements. For example, the decay $\mu \rightarrow 3 e$, while theoretically appealing, requires a triple coincidence and sensitivity to the whole phase space of the decay, making it experimentally quite challenging. It is the coherent muon to electron conversion, where with sufficient energy resolution on the conversion electron the signal can be resolved from background, that holds the most promise for many orders of magnitude improvement. Projected muon fluxes at present or proposed facilities will permit the experimental sensitivity to be pushed to the $10^{-16}$ to $10^{-18}$ level. Such a program has been proposed both for Fermilab (Mu2e)[42] and for J-PARC (COMET, PRISM-PRIME)[41]. At a neutrino factory, one might be able to reach the $10^{-19}$ to $10^{-20}$ level, or if the J-PARC or Fermilab experiments find a signal, explore in detail the nature of the lepton flavor violating interaction.

The muonium to antimuonium conversion (last process in Eq.3.1) represents a change of two units of lepton number, analogous to $K^{0} \bar{K}^{0}$ oscillations. This process was originally proposed by Pontecorvo[43]. An experiment at PSI[44] obtained a single event sensitivity of $P_{M \bar{M}}=8.2 \times$ $10^{-11}$ which implies a coupling $G_{M \bar{M}} \leq 3 \times 10^{-3} G_{F}$ at $90 \%$ C.L., where $G_{F}$ is the Fermi coupling constant. A broad range of speculative theories such as left-right symmetry, R-parity violating supersymmetry, etc.[45], could permit such an oscillation.

\section{Summary and Conclusions}

The program of muon physics discussed here provides compelling physics opportunities for the neutrino factory. The observation of a muon electric dipole moment, or a charged lepton flavor violation reaction would be as revolutionary as the discovery of neutrino mass has been. It is imperative that as the design of a neutrino factory goes forward, that every effort be made to design a facility that can support muon experiments, as well as the neutrino program. Acknowledgments:

I wish to thank my experimental friends, David Hertzog, Klaus Jungmann, Yoshi Kuno, Jim Miller and Yannis Semertzidis for many helpful and informative conversations. Thanks to my theoretical colleagues Andrzej Czarnecki, Sacha Davidson, John Ellis, André de Gouvêa, Bill Marciano and Eduardo de Rafael for useful conversations.

\section{References}

[1] See Figure 5 in Paul Kunze, Z. Phys. 83 (1933) 1.

[2] Carl D. Anderson and Seth H. Neddermeyer, Phys. Rev. 50 (1936) 263, and Seth H. Neddermeyer and Carl D. Anderson, Phys. Rev. 51 (1937) 844.

[3] ( $g-2)$ Collaboration: (a) R.M. Carey et al., Phys. Rev. Lett. 82 (1999) 1632; (b) H.N. Brown et al., Phys. Rev. D 62 (2001) 091101; (c) H.N. Brown, et al., Phys. Rev. Lett. 86 (2001) 2227; (d) G.W. 
Bennett, et al., Phys. Rev. Lett. 89, 101804 (2002); (e) G.W. Bennett, et al., Phys. Rev. Lett. 92 , 161802 (2004). (f) G.W. Bennett, et al., Phys. Rev. D 73 (2006) 072003.

[4] P.A.M. Dirac, Proc. R. Soc. (London) A117, 610 (1928), and A118, 351 (1928). See also, P.A.M. Dirac, The Principles of Quantum Mechanics, 4th edition, Oxford University Press, London, 1958. Eq. 2.1 uses Dirac's original notation.

[5] N.F. Ramsey Phys. Rev. 109 (1958) 225.

[6] L. Landau, Nucl. Phys. 3 (1957) 127.

[7] A. Angelopoulos, et al. (CPLEAR Collaboration), Phys. Lett. 444B (1998) 43.

[8] E.M. Purcell and N.F. Ramsey, Phys. Rev. 78(1950) 807.

[9] C.J. Baker, et al., Phys. Rev. Lett. 97 (2006) 131801.

[10] B.C. Regan, et al., Phys. Rev. Lett. 88 (2002) 071805-1.

[11] J. Bailey, et al., Nucl. Phys. B150 (1979) 1.

[12] M.V. Romalis, W.C. Griffith, J.P. Jacobs, and E.N. Fortson, Phys. Rev. Lett. 86 (2001) 2505.

[13] R.L. Garwin, L.M. Lederman, M. Weinrich, Phys. Rev. 105, 1415, (1957) and R.L. Garwin, D.P. Hutchinson, S. Penman and G. Shapiro, Phys. Rev. 118 (1959) 271.

[14] B. Lee Roberts, The Next-generation Muon $(g-2)$ Experiment, in proceedings of 10th International Workshop on Neutrino Factories, Super beams and Beta beams (NuFact08), June 30 - July 5 2008, Valencia, Spain PoS (NUFACT08) 102.

[15] J. Äystö, et al., hep-ph/0109217, 2001. This report of a CERN working group lays out a nice case for muon physics at a neutrino factory.

[16] Physics at a future Neutrion Factory and super-beam facility, S. King, K. Long, Y. Nagashima, B.L. Roberts and O. Yasuda editors, arXiv:0710.4947v2 [hep-ph], Nov. 2007.

[17] J. R. Ellis, J. Hisano, M. Raidal and Y. Shimizu, Phys. Lett. B 528 (2002) 86.

[18] J. R. Ellis and M. Raidal, Nucl. Phys. B 643, (2002) 229.

[19] K.S. Babu, B. Datta, and R.N. Mohapatra, Phys. Rev. Lett.85 (2000) 5064.

[20] J.L. Feng, K.T. Matchev .Y, Shadmi, Nucl. Phys. B 613, 366 (2001), and Phys. Lett. B555 (2003) 89.

[21] F.J.M. Farley, et al., Phys. Rev. Lett. 92 (2004) 052001.

[22] A. Adelmann and K. Kirsh, hep-ex/0606034, 2006.

[23] J-PARC Letter of Intent L22, January 2003 Search for a permanent muon electric dipole moment at the $10^{-24}$ e cm level., Y. Kuno, J.Miller, Y. Semertzidis spokespersons.

[24] N. Cabibbo, Phys. Rev. Lett. 10 (1963) 531.

[25] M. Kobayashi and T. Maskawa, Prog. Theor. Phys. Japan, 49 (1973) 652.

[26] Yoshitaka Kuno and Yasuhiro Okada, Rev. Mod. Phys. Phys. 73 (2001) 151, and references therein.

[27] R. Barbieri, L. J. Hall and A. Strumia, Nucl. Phys. B445, 219 (1995).

[28] K. Hasegawa, C.S. Lim, K. Ogure. Phys. Rev. D68, 053006 (2003).

[29] Ryuichiro Kitano, Phys. Lett. B481 (2000) 39.

[30] Ryuichiro Kitano, Masafumi Koike, Shinji Komine, Yasuhiro Okada, Phys. Lett. B575 (2003) 300. 
[31] Gabriela Barenboim, G.C. Branco, Andre de Gouvea, M.N. Rebelo Phys. Rev. D64 (2001) 073005.

[32] Andre de Gouvea, Smaragda Lola, Kazuhiro Tobe Phys. Rev. D63 (2001) 035004.

[33] V. Cirigliano, A. Kurylov, M.J. Ramsey-Musolf, P. Vogel Phys. Rev. D70 (2004) 075007.

[34] Francesca Borzumati, Antonio Masiero, Phys. Rev. Lett. 57 (1986) 961.

[35] P. Paradisi, hep-ph/0505046v2, J. High Energy Phys. 0510(2005) 006.

[36] Antonio Masiero, Sudhir K. Vempati and Oscar Vieves, New. J. Phys. 6 (2004) 421, hep-ph/0407325v2, and references therein.

[37] Seungwon Baek, Toru Goto, Yasuhiro Okada, Ken-ichi Okumura Phys. Rev. D64 (2001) 095001.

[38] A. Czarnecki, W.J. Marciano and K. Melnikov, hep-ph/9801218 AIP Conf. Proc. 435 (1998) 409.

[39] Ryuichiro Kitano, Masafumi Koike, Yasuhiro Okada, Phys. Rev. D66 (2002) 096002.

[40] The MEG Experiment at PSI. A. Baldini and T. Mori spokespersons. http://meg.web.psi.ch/ and S. Ritt, Nucl. Phys. B (Proc. Suppl.) 162 (2006) 279.

[41] J-PARC Letter of Intent L24, L25, January 2003 and the COMET proposal 1007, Y. Kuno Spokesperson.

[42] The Mu2e proposal to Fermilab, R. Bernstein and J. Miller co-spokespersons.

[43] B. Pontecorvo, Zh. Eksp. Teor. Fiz. 33 (1957) 549, [Sov. Phys. JETP 6 (1958) 429]; G. Feinberg and S. Weinberg, Phys. Rev. 123 (1961) 1439.

[44] L. Willmann, et al., Phys. Rev. Lett. 82 (1999) 49

[45] P. Herczeg and R.N. Mohapatra, Phys. Rev. Lett. 69 (1992) 2475; A. Halprin, Phys. Rev. Lett. 48 (1982) 1313; R.N. Mohapatra, Z. Phys. C56 (1992) 117; G.G. Wong and W.S. Hou, Phys. Rev. D50 (1994) R2962; A. Halprin and A. Masiero, Phys. Rev. D48 (1993) 2987; H. Fujii, Y. Mimura, K. Sasaki and T. Sasaki, Phys. Rev. D49 (1994) 559; P.H. Frampton and M. Harada, Phys. Rev. D58 (1998) 095013; V. Pleitez, Phys. Rev. D61 (2000) 057903; G. Cvetic, et al., hep-ph/0504126. 